This item was submitted to Loughborough's Research Repository by the author.

Items in Figshare are protected by copyright, with all rights reserved, unless otherwise indicated.

\title{
Can shoulder range of movement be measured accurately using the Microsoft Kinect sensor plus Medical Interactive Recovery Assistant software?
}

\section{PLEASE CITE THE PUBLISHED VERSION}

https://doi.org/10.1016/j.jse.2017.06.004

\section{PUBLISHER}

(c) Journal of Shoulder and Elbow Surgery Board of Trustees. Published by Elsevier

VERSION

AM (Accepted Manuscript)

\section{PUBLISHER STATEMENT}

This work is made available according to the conditions of the Creative Commons Attribution-NonCommercialNoDerivatives 4.0 International (CC BY-NC-ND 4.0) licence. Full details of this licence are available at: https://creativecommons.org/licenses/by-nc-nd/4.0/

\section{LICENCE}

CC BY-NC-ND 4.0

\section{REPOSITORY RECORD}

Wilson, James D., Jennifer Khan-Perez, Dominic Marley, Susan Buttress, Michael Walton, Baihua Li, and Bibhas Roy. 2019. "Can Shoulder Range of Movement Be Measured Accurately Using the Microsoft Kinect Sensor Plus Medical Interactive Recovery Assistant Software?". figshare. https://hdl.handle.net/2134/33761. 


\title{
Can shoulder range of movement be measured accurately using the Microsoft Kinect sensor plus Medical Interactive Recovery Assistant (MIRA) software?
}

\author{
James D. Wilson, FRCS(TrOrth) ${ }^{a, *}$, Jennifer Khan-Perez, MBBS ${ }^{b}$, Dominic Marley, \\ MBBS $^{c}$, Susan Buttress, MPhil ${ }^{d}$, Michael Walton, FRCS(TrOrth) ${ }^{\mathrm{e}}$, Baihua Li, PhD \\ Bibhas Roy, FRCS(TrOrth) ${ }^{g}$
}

\author{
${ }^{a}$ Trauma and Orthopaedics Department, Bolton National Health Service Foundation Trust, Manchester, UK

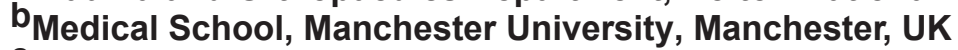

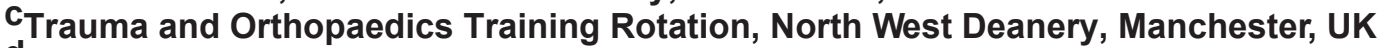 \\ d School of Health Sciences, Salford University, Salford, UK

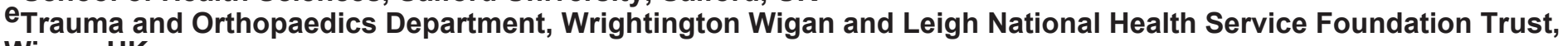 \\ Wigan, UK \\ ${ }^{f}$ Computer Science Department, Loughborough University, Loughborough, UK \\ 9Trauma and Orthopaedics Department, Central Manchester University, Hospitals National Health Service \\ Foundation Trust, Manchester UK
}

Background:: This study compared the accuracy of measuring shoulder range of movement (ROM) with a simple laptop-sensor combination vs. trained observers (shoulder physiotherapists and shoulder sur-geons) using motion capture (MoCap) laboratory equipment as the gold standard.

Methods: The Microsoft Kinect sensor (Microsoft Corp., Redmond, WA, USA) tracks 3-dimensional human motion. Ordinarily used with an Xbox (Microsoft Corp.) video game console, Medical Interactive Recov-ery Assistant (MIRA) software (MIRA Rehab Ltd., London, UK) allows this small sensor to measure shoulder movement with a standard computer. Shoulder movements of 49 healthy volunteers were simultaneously mea-sured by trained observers, MoCap, and the MIRA device. Internal rotation was assessed with the shoulder abducted $90^{\circ}$ and external rotation with the shoulder adducted. Visual estimation and MIRA measurements were compared with gold standard MoCap measurements for agreement using Bland-Altman methods. Results: There were 1670 measurements analyzed. The MIRA evaluations of all 4 cardinal shoulder move-ments were significantly more precise, with narrower limits of agreement, than the measurements of trained observers. MIRA achieved $\pm 11^{\circ}\left(95 \%\right.$ confidence interval $\left.[\mathrm{Cl}], 8.7^{\circ}-12.6^{\circ}\right)$ for forward flexion vs. $\pm 16^{\circ}(95 \%$ Cl, $\left.14.6^{\circ}-17.6^{\circ}\right)$ by trained observers. For abduction, MIRA showed $\pm 11^{\circ}\left(95 \% \mathrm{Cl}, 8.7^{\circ}-12.8^{\circ}\right)$ against 
Accurate measurement of shoulder range of movement (ROM) is an extremely important element of assessing shoul-der pathology. Restriction of shoulder movement limits function. Identifying impaired ROM aids diagnosis and eval-uation of severity for common conditions such as frozen shoulder, rotator cuff deficiency, and subacromial impinge-ment. Repeat measurement can help to track a patient's recovery or response to treatment. ${ }^{3,13}$

Various methods of measurement are used to help manage shoulder rehabilitation, including the traditional goniometer, questionnaires, ${ }^{13}$ camcorders, electromagnetic sensors, ${ }^{15,16}$ and visual estimation. ${ }^{12}$ An accurate, automated process may be more efficient and objective compared with traditional assessments. ${ }^{12,14}$ The use of remote sensor technology provides several advan-tages over conventional modes of measurement. It delivers increased capacity for quantification of motor performance and real-time feedback enhancing patient motivation. ${ }^{10}$

The Kinect sensor (Microsoft Corp., Redmond, WA, USA) was developed as an add-on for the Xbox 360 video game console (Microsoft Corp.). It allows users to interact with the gaming system by tracking body movement in 3 dimen-sions (3D). The key to the 3-D movement recognition is the sensor's depth camera, which uses an infrared (IR) laser pro-jector that generates a speckle pattern and an IR camera that detects the reflections from objects. The Kinect is able to create a 3-D map of these objects by measuring deformations in the reference speckle pattern. ${ }^{7}$ The Kinect sensor can be paired with a standard notebook or desktop computer and software developed to measure and track body movements; for example, as part of an in-home shoulder rehabilitation program.

The current gold standard method of measuring body move-ment is by using motion capture (MoCap) technology such as the Vicon (Vicon Motion Systems Ltd., Oxford, UK) markertracking system. It incorporates multiple high-resolution cameras and makes use of IR reflective markers to achieve millimeter resolution of 3-D spacial displace-ments at greater than 100 frames per second. ${ }^{7,11}$ Conversely, clinical assessment and rehabilitation of patents with shoul-der pathology is routinely performed by trained observers who visually assess shoulder movement without the aid of sensors or MoCap technology.

This study assessed the accuracy of the Kinect sensor paired with the software developed by Medical Interactive Recov-ery Assistant (MIRA; MIRA Rehab Ltd., London, UK). Therefore, the level of agreement between MoCap and the
Kinect + MIRA system was compared with the level of agreement between MoCap and the visual estimation of trained observers.

\section{Materials and methods}

The investigation was conducted in the dedicated MoCap laboratory at the Institute for Biomedical Research into Human Movement. This MoCap laboratory in Manchester University uses a Vicon system consisting of 10 high-resolution cameras fitted with IR optical filters and an array of IR light-emitting diodes for illu-mination. The cameras were calibrated before the MoCap session. The calibration accuracy achieved was $0.01 \mathrm{~mm}$. Forty-nine healthy individuals consented to participate. Reflective markers were applied to the participants in a standardized pattern, based on surface an-atomic locations (plug-in-gait model upper limbs and thorax; Fig. 1). Using the 3-D locations of these markers recorded by the system in real-time, the Vicon software calculated joint centers and com-posite shoulder movement relative to the thorax (scapula-thoracic and glenohumeral motions combined).

The Kinect sensor was set up within the MoCap laboratory to facilitate simultaneous measurements. The sensor was placed $1.5 \mathrm{~m}$ above the floor on a tripod, 2 to $3 \mathrm{~m}$ from the participant (Fig. 2). A Universal Serial Bus 2.0 port was used to connect the Kinect sensor

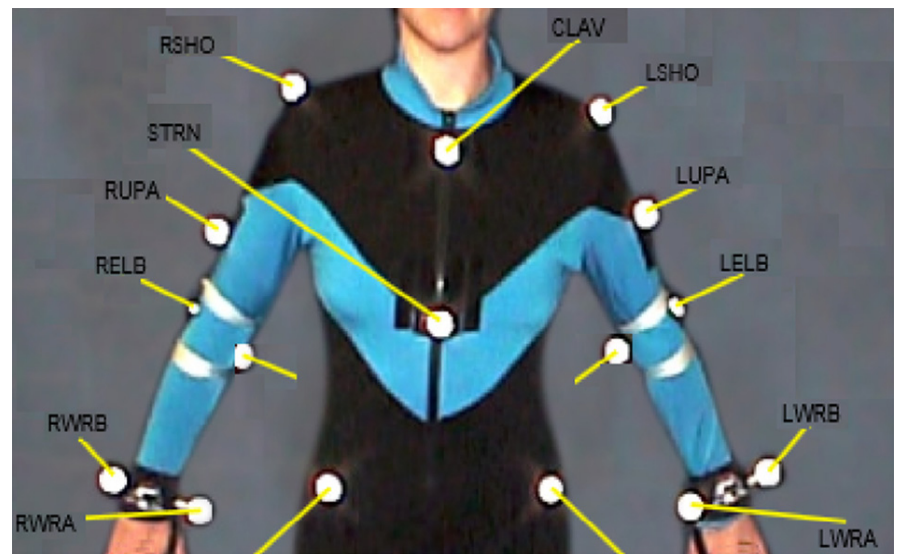

Figure 1 Positions of infrared reflective markers. The 4 addi-tional markers without labels are right and left elbow A and right and left anterior superior iliac spine. CLAV, medial clavicle; $L E L B$, left elbow B; $L S H O$, left shoulder; $L U P A$, left upper arm; $L W R A$, left wrist $\mathrm{A} ; \angle W R B$ left wrist $\mathrm{B}$; $R E L B$, right elbow $\mathrm{B} ; R S H O$, right shoulder; $R U P A$, right upper arm; $R W R A$, right wrist $\mathrm{A} ; R W R B$, right wrist B; STRN, sternum. 


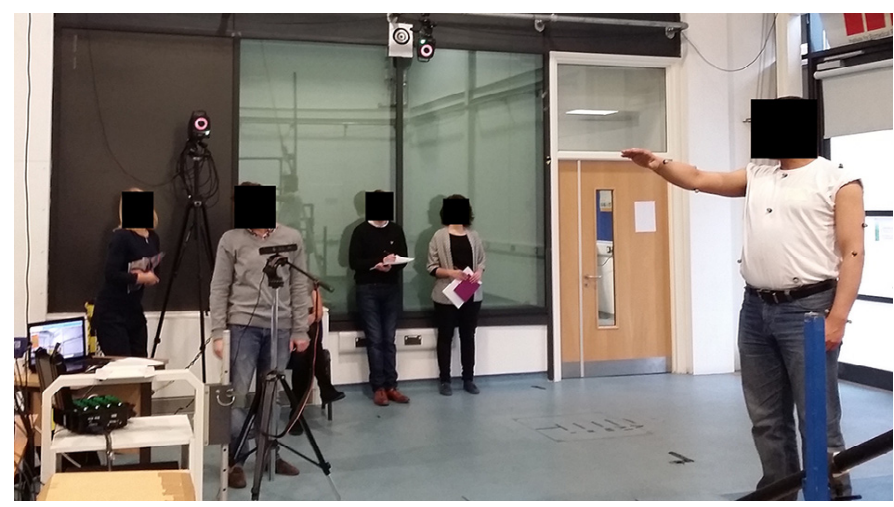

Figure 2 Study set-up showing motion capture cameras, Medical Interactive Recovery Assistant (MIRA) software (MIRA Rehab Ltd., London, UK)/Kinect (Microsoft Corp., Redmond, WA, USA) sensor, and trained observers.

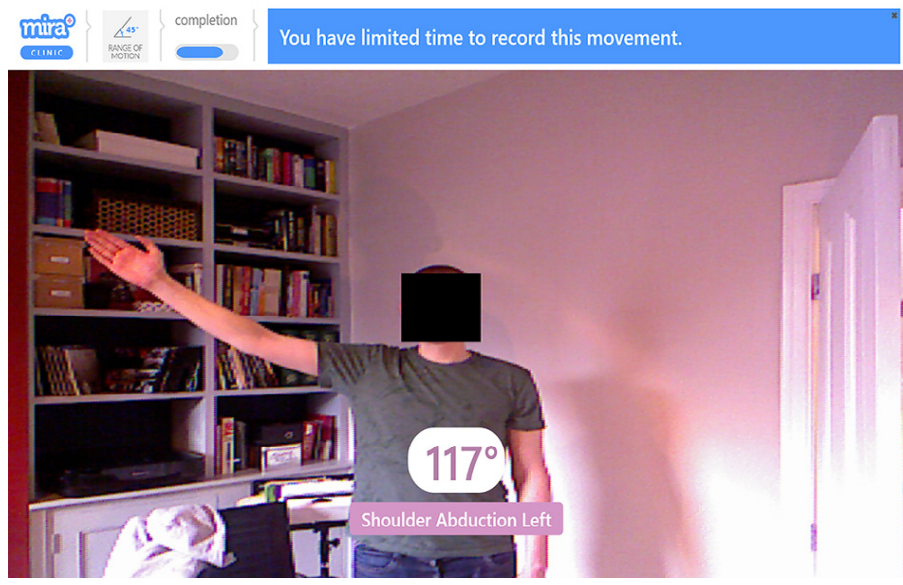

Figure 3 Screen shot of Medical Interactive Recovery Assistant (MIRA) software (MIRA Rehab Ltd., London, UK) displaying mea-sured abduction angle using the Kinect (Microsoft Corp., Redmond, WA, USA) sensor. (The screen is set up to act as a mirror for a more intuitive user experience, hence the apparent leftright discrepancy).

to a Lenovo (Beijing, China) laptop with a Windows 7 (Microsoft Corp.), 64-bit operating system, $8 \mathrm{~GB}$ of random access memory, and a 2.3-GHz Intel i7 (Intel Corp., Santa Clara, CA, USA) pro-cessor. MIRA software was used to process the Kinect data. This was achieved using the Microsoft Kinect Software Development Kit (SDK) 1.8 for Windows and the ROM measurement tool that is part of the MIRA 1.3 platform software installed on the laptop. MIRA calculated the 3D angle of composite shoulder movement and dis-played the result in real time (Fig. 3).

In addition to the Vicon system and the Kinect + MIRA pairing, trained observers also recorded their visual assessment of the angle of shoulder movement. These observers comprised 1 consultant orthopedic surgeon, 2 orthopedic specialty trainee registrars, 3 upper limb specialist physiotherapists, and 1 medical student who had received specific training in shoulder ROM assessment for this project. As in a standard clinical setting, the observers were allowed to move around the participants to view them from differ-ent positions while estimating the shoulder movement angles, without additional equipment such as goniometers. This was to emulate routine clinical practice and to allow multiple simultaneous obser-vations to be made.

The participants were asked to perform each of the 4 cardinal shoulder movements: abduction, forward flexion, and internal and external rotation. External rotations were performed with the shoul-der adducted. Internal rotation was performed with the shoulder abducted at $90^{\circ}$. This abducted position was used so that the par-ticipant's trunk did not act as a block to full internal rotation. Each movement was then held in a static position to allow simultaneous measurement by the Kinect + MIRA system, the MoCap, and the trained observers. To assess agreement across the full spread of pos-sible measurements, the participants were asked to move their shoulder to the full extent of its range and then were asked to repeat the move-ment to a point short of full range. This second position was chosen by the participant to help generate a pseudorandom spread of dif-ferent shoulder positions. The measurements were performed for both shoulders of each participant.

The trained observers were blinded to the measurements of the other observers and of the Kinect + MIRA system. The data from the MoCap system required extensive processing, which was per-formed over several days after completion of the study. This analysis allowed calculation of the movement angles. This was done in iso-lation from the Kinect + MIRA operator and the group of trained observers. Therefore, there was no possibility of the measure-ments from the trained observers or the Kinect + MIRA system being biased or influenced by the measurements the MoCap system recorded.

\section{Statistical analysis}

Agreements between the gold standard (MoCap) and the other 2 methods of measurement (Kinect + MIRA and estimation by trained observers) were analyzed as recommended by Bland and Altman. ${ }^{1,2}$ This comprised calculating the difference from the MoCap mea-surement for every Kinect + MIRA measurement and each trained observer measurement. The spread of these differences was then tested for normality using the Shapiro-Wilk calculation. Correla-tions were assessed using the Pearson test or the Spearman test if a normal distribution was not present. Scatter plots of these corre-lations were then generated. Agreement between the measurements methods were demonstrated using Bland-Altman plots. The limits of agreement (LOA) were calculated, and the 95\% confidence intervals (CIs) of the LOA were also calculated. These analyses were performed using IBM SPSS Statistics 22 software (IBM, Armonk, NY, USA).

\section{Results}

There were 1670 measurements available for analysis from the observers, MoCap, and MIRA + Kinect systems. The anal-ysis had to exclude 49 measurements from the MoCap system because interference with IR reflections prevented calcula-tion of the angle of shoulder movement. However, only 1 MIRA + Kinect measurement was identified as an outlier re-quiring exclusion, and no measurements recorded by the trained observers required exclusion.

Correlations between the MoCap measurements and the other modes of measurement are reported in Table I. Scatter plots were also produced for comparison of the correlations with MoCap measurements (Fig. 4). These charts demonstrate 
Table I Correlation coefficients with motion capture for the cardinal shoulder movements

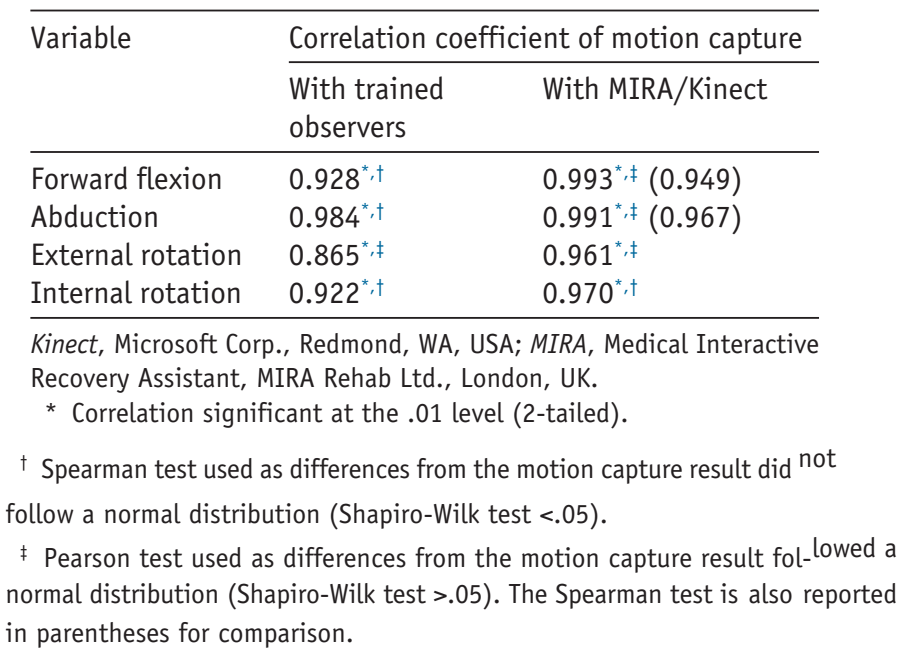

Table II Limits of agreement of the $\mathbf{2}$ measurement methods with motion capture for the cardinal shoulder movements

\begin{tabular}{|c|c|c|c|c|}
\hline \multirow[t]{2}{*}{ Variable } & \multicolumn{2}{|c|}{ Trained observer } & \multicolumn{2}{|c|}{ MIRA/Kinect } \\
\hline & $\begin{array}{l}\text { Limits of } \\
\text { agreement } \\
\left({ }^{\circ}\right)\end{array}$ & $\begin{array}{l}95 \% \\
\mathrm{CI}\left({ }^{\circ}\right)\end{array}$ & $\begin{array}{l}\text { Limits of } \\
\text { agreement } \\
\left({ }^{\circ}\right)\end{array}$ & $\begin{array}{l}95 \% \\
\mathrm{CI}\left({ }^{\circ}\right)\end{array}$ \\
\hline Forward flexion & 16 & $14.6-17.6$ & 11 & $8.7-12.6$ \\
\hline Abduction & 15 & $13.4-16.2$ & 11 & $8.7-12.8$ \\
\hline External rotation & 21 & $18.7-22.6$ & 10 & 8.1-11.9 \\
\hline Internal rotation & 18 & $16.0-19.3$ & 9 & $7.2-10.4$ \\
\hline
\end{tabular}

a much closer correlation with the MoCap measurements for the MIRA + Kinect system compared with the trained ob-server measurements.

To analyze the different measurement techniques further, the agreement was assessed using Bland-Altman plots (Fig. 5) and calculation of the LOA., ${ }^{1,2}$ These charts indicate the mean difference from the MoCap measurements as the solid black line, with the $95 \% \mathrm{CI}$ of this value shown by the solid red lines above and below it. The LOA are represented by the dotted black lines above and below the mean. The 95\% CIs of these values were also calculated and are shown as the dotted green lines. The LOA and their 95\% CIs are pre-sented in Table II for further clarity.

These Bland-Altman charts show that for each of the cardinal shoulder movements, the MIRA + Kinect system has a narrower spread of plots compared with the results from the trained observers. The calculated LOA show that the MIRA + Kinect has closer agreement to the MoCap than the trained observers. Furthermore, the $95 \%$ CIs of these values do not overlap, indicating that the superior agreement that has been found is statistically significant. The values pre-sented in Table II show how closely each measurement method agrees with the gold standard MoCap measurement. For example, $95 \%$ of the measurements performed by trained ob-servers for forward flexion should lie within $\pm 16^{\circ}$ of the MoCap measurement. However, the MIRA + Kinect system agrees more closely with $95 \%$ of measurements, lying within $\pm 11^{\circ}$.

\section{Discussion}

The power to measure and monitor shoulder movement using a portable and inexpensive sensor-based system brings po-tential benefits to research and clinical applications across a wide range of patient populations. ${ }^{5,8} \mathrm{We}$ compared both mea-surement techniques to the Vicon MoCap system because this is considered the gold standard for measurement accuracy. The MIRA + Kinect system carries benefits and drawbacks compared with other practical aspects of MoCap. Beyond the benefits of lower cost and portability, the MIRA + Kinect system does not require markers placed on the skin, which avoids lengthy set up time, potentially indelicate body exposure, and problems with inaccurate marker position. ${ }^{5}$

Vicon MoCap technology is vulnerable to interference with data collection caused by reflections from extraneous objects within the field of view or loss of signal from the IR reflec-tive markers. The trained observers were asked to remove jewelry that could reflect IR and to avoid standing in posi-tions that would block the line-of-sight of the MoCap cameras. However, despite these procedures and despite conducting the study in a dedicated MoCap laboratory with 10 high- reso-lution cameras, 49 measurements (13\%) were still lost to analysis because interference precluded calculation of the shoulder movement angles. This demonstrates that regard-less of the superior accuracy of the Vicon MoCap system, it faces limitations even when conditions are optimized and would not be a practical technology to apply within a clin-ical or home setting with limited space, multiple background objects, and people moving around. ${ }^{7}$

In contrast to this, we found that the MIRA + Kinect system would be more appropriate for use in a clinical or home-based rehabilitation role because it only produced 1 outlying data point $(0.3 \%)$ necessitating exclusion. It also uses sensors specifically designed to work within the home environment. ${ }^{7}$ However, it is important to note that the ability of the Vicon MoCap system to differentiate between scapulothoracic and glenohumeral components of shoulder movement is not emu-lated by the MIRA + Kinect system because only composite shoulder movement can be measured.

This study has demonstrated that the MIRA + Kinect system has better agreement with gold standard measurement com-pared with estimation by trained observers. However, there are other aspects to consider. Additional benefits of the system over trained observers is the objective nature of the sensor technol-ogy. The system can provide unbiased measurement for comparison between groups or before and after an interven-tion for the same individuals. Furthermore, although the system does involve a laptop and a Kinect sensor, its operation does 
Trained Observer Measurements
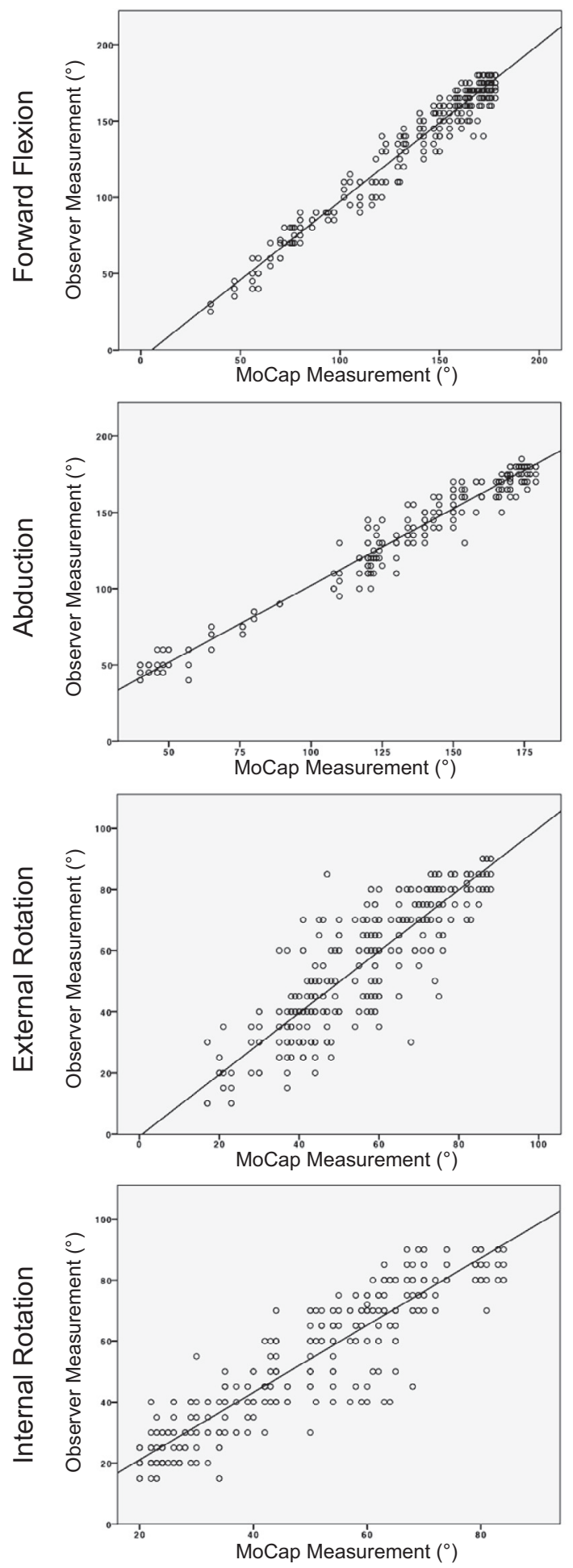

MIRA / Kinect Measurements


Figure 4 Scatter plots of measurement methods against motion capture (MoCap) for cardinal shoulder movements. MIRA, Medical Interactive Recovery Assistant, MIRA Rehab Ltd., London, UK; Kinect, Microsoft Corp., Redmond, WA, USA. 
Trained Observer Measurements
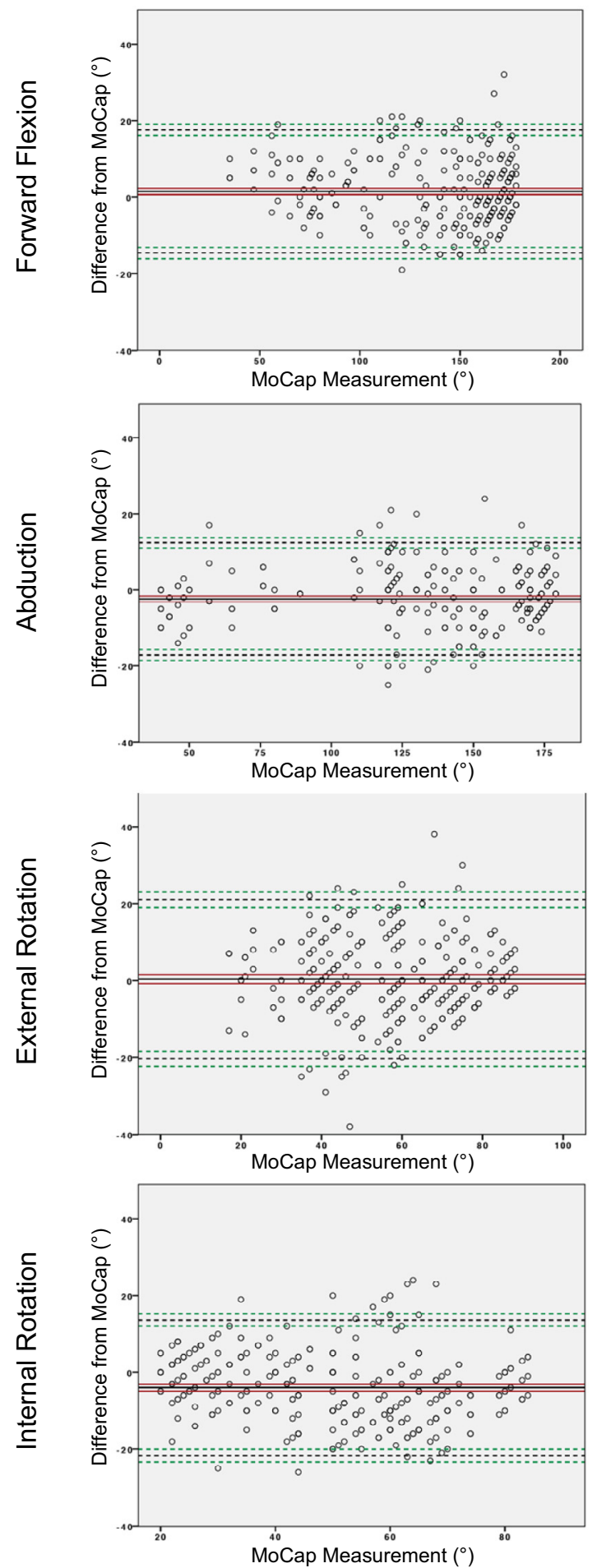

MIRA / Kinect Measurements
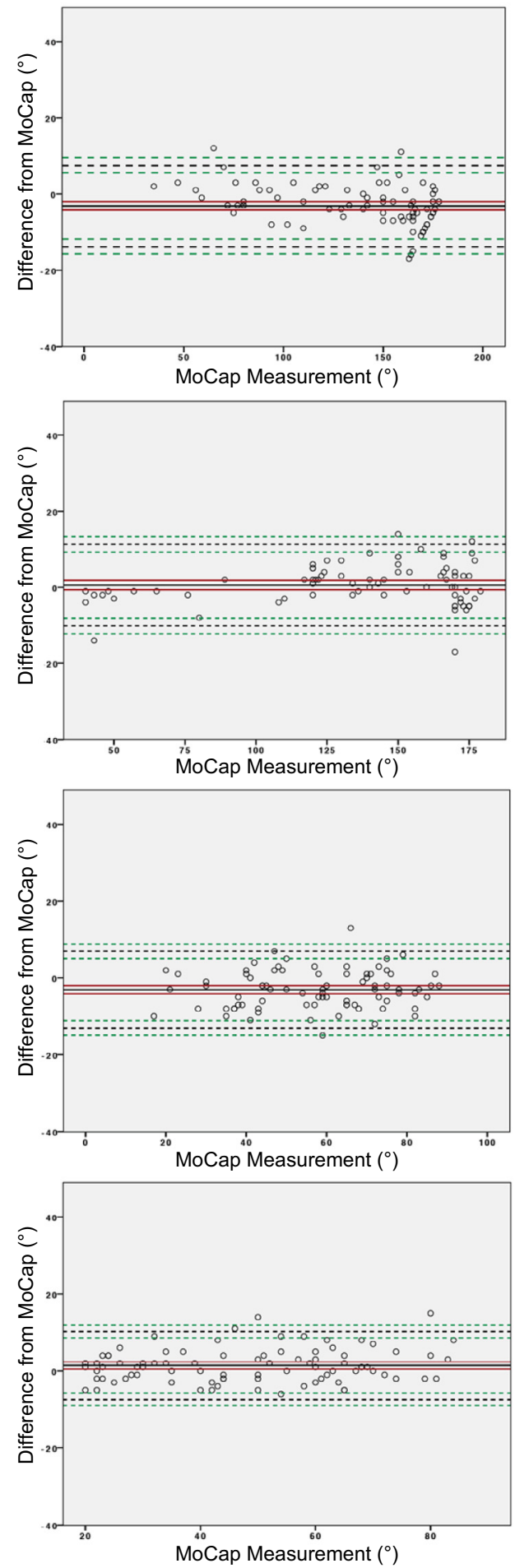

Figure 5 Bland-Altman Plots of measurement methods against motion capture (MoCap) for cardinal shoulder movements. The solid black line is the mean difference from the MoCap measurements, and the solid red lines indicate the $95 \%$ confidence interval of this value. The dotted black lines show the limits of agreement, with the dotted green lines indicating the $95 \%$ confidence intervals. 
not require a trained professional such as a specialist physio-therapist or orthopedic surgeon. Individuals can operate the system without supervision by following on-screen directions.

The utility of the Kinect sensor has been evaluated for joint position ${ }^{17}$ movement analysis in postural control and gait retraining, ${ }^{4-6}$ use in people with Parkinson disease, ${ }^{9}$ and authors have concluded that the sensor is useful as a means of measuring gross body movements and ergonomic assessments in "the field." $\mathrm{Xu}$ and McGorry ${ }^{17}$ found that the Kinect did not ac-curately identify joint centers, particularly for lower limb joints, but did comment that joint angle measurement may be of greater interest for occupational tasks than pure joint location.

Other authors have also assessed use of a Kinect sensor to specifically measure shoulder movement. Huber et $\mathrm{al}^{10}$ compared the Kinect sensor to goniometer measurement and to electromagnetic motion tracking sensors. They found increased levels of bias and broader limits of agreement. The MIRA software used with the Kinect sensor in our study has undergone several cycles of refinement to opti-mize the positioning of the participants and the movements they perform, which may account for the different conclu-sion. In addition, we compared the MIRA + Kinect system with the performance of trained observers, emulating routine clinical practice, rather than taking $5^{\circ}$ as an arbitrary target value.

Use of a sensor-based technology to measure shoulder movement has clear utility in providing objective data for re-search applications. It is unlikely to replace clinician observation to estimate shoulder ROM during routine clin-ical assessment, but it opens the possibilities of using this system to enhance rehabilitation protocols. Patients can be encouraged to engage with their shoulder exercise program by harnessing the principles of gamification within tailored "Exergames." Having good evidence that this technology can accurately track shoulder movement is a necessity before cli-nicians should consider its implementation.

Other novel technologies, such as wearable sensors, are being developed, also with the aim of accurately plotting body move-ments and using them for shoulder rehabilitation. ${ }^{12}$ Furthermore, an updated Kinect sensor (Kinect 2) offers increased sensitiv-ity and the potential for motion analysis of smaller joints. $^{5,17}$

We acknowledge that our study has several limitations. The participants were all asked to wear close-fitting garments to facilitate IR marker positioning. This is also beneficial for the Kinect sensor. We have not compared the different measure-ment methods for situations where loose or baggy clothing is being worn. Clinical examination is normally performed with appropriate exposure of the limb, but if the sensor is being used in a home setting, a participant may not remove baggy garments for the assessment.

The measurement of internal rotation was performed with the participant abducting the shoulder to $90^{\circ}$. This position was used so that the forearm can move freely, without contacting the participant's abdomen. This position of abduction may be difficult to reach for some individuals with shoulder pathology.

Performing Vicon and MIRA + Kinect measurements si- distort the 3-D model generated by the IR Kinect sensor. However, we found the model had good agreement with the Vicon system, and other authors have found this effect to be minimal. ${ }^{5}$

The participants did not have shoulder pathology and, there-fore, do not represent the population that would be using such a system. This did, however, allow testing across the full range of possible shoulder movements, unconstrained by pain or stiffness. Our measurements were also taken with the par-ticipants in a static pose. This was to facilitate simultaneous measurement by the MoCap, MIRA + Kinect, and the trained observers. Therefore, our results may not be applicable to dynamic shoulder movements, and further validation of this aspect may be required.

\section{Conclusion}

MIRA software paired with a Kinect sensor measures all cardinal shoulder movements with significantly closer agreement to Vicon MoCap than trained observer measurements. Therefore, use of this system to measure shoulder movement during shoulder assessment and re-habilitation would be acceptable. In addition, this technology may allow precise shoulder ROM measure-ment outside the clinic setting.

\section{Disclaimer}

Bibhas Roy has been a clinical advisor to MIRA Rehab since 2013. This has helped shape the exergames devel-opment by providing clinical context. He has not received any direct financial benefit from MIRA Rehab and has not invested money in the company but does hold share options in the company. All of the other authors, their immediate families, and any research foundations with which they are affiliated have not received any financial payments or other benefits from any commercial entity related to the subject of this article.

Central Manchester University Hospital has a revenue sharing agreement with MIRA Rehab. MIRA Rehab has financially benefited from Central Manchester University Hospitals NHS Foundation Trust due to a project-specific grant.

\section{References}

1. Bland JM, Altman DG. Statistical methods for assessing agreement between two methods of clinical measurement. Lancet 1986;1:307-10.

2. Bland JM, Altman DG. Measuring agreement in method comparison studies. Stat Methods Med Res 1999;8:135-60.

3. Bunker T. (ii) Frozen shoulder. Orthop Trauma 2011;25:11-8. http://dx.doi.org/10.1016/j.mporth.2011.01.007 
4. Clark RA, Pua YH, Bryant AL, Hunt MA. Validity of the Microsoft Kinect for providing lateral trunk lean feedback during gait retraining. Gait Posture 2013;38:1064-6. http://

dx.doi.org/10.1016/j.gaitpost .2013.03.029

5. Clark RA, Pua YH, Fortin K, Ritchie C, Webster KE, Denehy L, et al. Validity of the Microsoft Kinect for assessment of postural control. Gait Posture 2012;36:372-7. http://dx.doi.org/10.1016/ j.gaitpost.2012.03.033

6. Clark RA, Pua YH, Oliveira CC, Bower KJ, Thilarajah S, McGaw R, et al. Reliability and concurrent validity of the Microsoft Xbox One Kinect for assessment of standing balance and postural control. Gait Posture 2015;42:210-3. http://dx.doi.org/10.1016/ j.gaitpost.2015.03.005

7. Dutta T. Evaluation of the Kinect sensor for 3-D kinematic measurement in the workplace. Appl Ergon 2012;43:645-9.

8. Fernández-Baena A, Susin A, Lligadas X. Biomechanical validation of upper-body and lower-body joint movements of kinect motion capture data for rehabilitation treatments. Presented at the 2012 Fourth International Conference on Intelligent Networking and Collaborative Systems, Bucharest, Romania, Sept 19-21, 2012. IEEE. 2012. p. 656-61. http://dx.doi.org/10.1109/iNCoS.2012.66

9. Galna B, Barry G, Jackson D, Mhiripiri D, Olivier P, Rochester L. Accuracy of the Microsoft Kinect sensor for measuring movement in people with Parkinson's disease. Gait Posture 2014;39:1062-8. http://dx .doi.org/10.1016/j.gaitpost.2014.01.008

10. Huber ME, Seitz AL, Leeser M, Sternad D. Validity and reliability of Kinect skeleton for measuring shoulder joint angles: a feasibility study. Physiotherapy 2015;101:389-93. http://dx.doi.org/10.1016/ j.physio .2015.02.002

11. Leightley D, Darby J, Li B, McPhee JS, Yap MH. Human activity recognition for physical rehabilitation. Presented at the 2013 IEEE International Conference Systems, Man, and Cybernetics-SMC Manchester, UK, Oct 13-16 2013. http://dx.doi.org/10.1109/ SMC.2013.51

12. Lin HC, Chiang SY, Lee K, Kan YC. An activity recognition model using inertial sensor nodes in a wireless sensor network for frozen shoulder rehabilitation exercises. Sensors (Basel) 2015;15:2181-204. http://dx.doi.org/10.3390/s150102181

13. Mao CY, Jaw WC, Cheng HC. Frozen shoulder: correlation between the response to physical therapy and follow-up shoulder arthrography. Arch Phys Med Rehabil 1997;78:857-9.

14. Maund E, Craig D, Suekarran S, Neilson AR, Wright K, Brealey S, et al. Management of frozen shoulder: a systematic review and costeffectiveness analysis. Health Technol Assess 2012;16:http:// dx.doi.org/ 10.3310/hta16110

15. Rundquist PJ, Anderson DD, Guanche CA, Ludewig PM. Shoulder kinematics in subjects with frozen shoulder. Arch Phys Med Rehabil 2003;84:1473-9. http://dx.doi.org/10.1016/S0003-9993(03) 00359-9

16. Walther M, Werner A, Stahlschmidt T, Woelfel R, Gohlke F. The subacromial impingement syndrome of the shoulder treated by conventional physiotherapy, self-training, and a shoulder brace: results of a prospective, randomized study. J Shoulder Elbow Surg 2004;13:417-23.

17. Xu X, McGorry RW. The validity of the first and second generation Microsoft Kinect ${ }^{\mathrm{TM}}$ for identifying joint center locations during static postures. Appl Ergon 2015;49:47-54. http://dx.doi.org/10.1016/ j.apergo.2015.01.005 